\title{
Occupational Health Risk among Selected Cement Factory Workers in Dang District of Nepal
}

\author{
Pawan Pandeya ${ }^{1}$, Durga Khadka Mishra ${ }^{1}$, Shankar Prasad Khanal ${ }^{1,2}$, \\ Yadav Prasad Joshi ${ }^{1,5}$, Sujan Babu Marahatta ${ }^{1,5,6}$, Arzoo Neupane ${ }^{3}$, \\ Rashmita Chhetri $^{1}$, Santaram Chaudhari ${ }^{4}$, Sushila Baral ${ }^{1}$, Neha Deo ${ }^{1}$ \\ ${ }^{1}$ Manmohan Memorial Institute of Health Sciences, Soalteemode, Kathmandu \\ ${ }^{2}$ Central Department of Statistics, Tribhuvan University Nepal \\ ${ }^{3}$ Sahid Gangalal Memorial Heart Center, Bansbari, Kathmandu \\ ${ }^{4}$ Maharajgunj Medical College, Institute of Medicine, Tribhuvan University Nepal \\ ${ }^{5}$ Faculty of Health Science and Technology, Nepal Open University, Lalitpur, Nepal \\ ${ }^{6}$ Health Research Together Initiative-Nepal (HeaRT: Initiative), Kathmandu, Nepal
}

Corresponding Author: Pawan Pandeya

\begin{abstract}
Background: The health risks was reported in the cement industry in various study conducted in different settings. This study aimed to assess the occupational health risks among cement factory workers of Dang district of Nepal.

Methods: A cross-sectional study in 212 workers was conducted in Samrat Cement Company and Sonapur Minerals and Oils of Dang district in between July, 2019 to January 2020 using structured questionnaire. Proportionate stratified random sampling was used for participant selection. The collected information was entered in Statistical Package for Social Sciences (SPSS). Descriptive and inferential statistical analysis was performed.

Results: Of 212 workers, the respiratory and musculoskeletal health risks were reported by $13.2 \%$ and $22.2 \%$ of factory workers, respectively. About $29.2 \%$ were smokers. Two-third (66\%) had poor knowledge of Occupational Health and Safety related questions and $17.9 \%$ had poor practice to prevent respiratory problems. Smokers $(\mathrm{p}=0.004)$ and access to safety measures $(\mathrm{p}=0.008)$ were significantly associated with respiratory problems. Educational status $(\mathrm{p}=0.021)$, work shift $(\mathrm{p}=0.022)$ and first aid availability $(\mathrm{p}=0.009)$ were statistically significant with musculoskeletal health risk.

Conclusion: Low occupational health risk and low knowledge was reported by cement factory workers. Special protection to workers above 40 years and declaring the cement factory as smoking restricted area could minimize respiratory risk. Access to and condition of safety devices need to be monitored by factory administration. Behavior change communication and safety orientation could help the workers use safety devices.
\end{abstract}

Keywords: Occupational health risk, respiratory health risk, musculoskeletal health risk, knowledge, practice, cement factory workers.

\section{INTRODUCTION}

Occupational health and safety promotes the health and well-being of workers through anticipation, recognition, evaluation and control of hazards arising in/from the workplace ${ }^{(1)}$.

Occupation health protects, promotes the worker's health either by preventing or controlling occupational accidents and diseases by reducing or eliminating factors or conditions hazardous to health and safety at work. ${ }^{(2)}$.

In developing countries, though on an average, one-third of the lifetime of workers is spent in the workplace the working condition is very poor due to lack 
of knowledge, attitude and behavior among employees, employers and regulating bodies (3). Exposure to cement reveals the complaint of the respiratory tract in high frequency among loading workers with persistent cough $54 \%$ and breathlessness $47 \%^{(4)}$.

The common causes of accident in cement factories include; accident due to machinery, accident due to manual material handling, accident due to electricity, accident due to lack of PPE, accident due to occupational stress, accident due to lack of safety culture, fire, etc. ${ }^{(5)}$.

Emissions from cement industries are degrading human health by deteriorating air quality ${ }^{(6)}$. Human health is adversely affected by the emissions that occur from cement industries in a variety of ways, like itchy eyes, respiratory diseases like tuberculosis, chest discomfort, chronic bronchitis, asthma attacks, cardiovascular diseases and even premature death ${ }^{(7)}$. Likewise, cement exposure has a high risk of developing chronic respiratory symptoms and COPD $^{(8)}$.

Worldwide, the problem of occupational safety can be observed. In the United Arab Emirates (UAE), although $74.5 \%$ had knowledge that dust exposure results in serious health hazards while only $28.8 \%$ used masks all the time during working hours ${ }^{(9)}$.

According to International Labor Organization (ILO) estimates, throughout the world, there are over 300 million work accidents (both fatal and non-fatal), about 160 million work disease cases every year and some 2.3 million persons die from work-related accidents and diseases ${ }^{(10)}$.

In Northwest Ethiopia workers working in night shifts were two times more likely to develop respiratory symptoms than do not work the night shift. Workers with service year more than 5 years had likely to develop respiratory symptoms. Training on occupational health and safety-related to dust health effects could reduce respiratory symptoms by $82 \%$. Also, the occupational history of dust exposure is likely to develop respiratory symptoms as per a study in Northwest Ethiopia ${ }^{(11)}$.

OHS concept is still new in Nepal because of high illiteracy among workers, lack of awareness, the poor performance of the concerned government authorities in implementation. In Nepal, in some workplaces, personal protective equipment is found to be provided by management but due to no or low use of them, management did not provide such PPE later on. Lack of awareness and felt inconvenience by workers are the major cause of not using $\mathrm{PPE}^{(12)}$.

In Nepal, cement production is growing steadily over the past few years ${ }^{(13)}$. At present in Nepal, around 59 cement industries under the private sector and two governments owned cement industry have been registered in the department of the industry which are in operation ${ }^{(5)}$. National policy and legislation are not adequate in Nepal. Moreover, the working environment is not yet being labor-friendly ${ }^{(14)}$.

Study on Maruti cement factory workers reflected that $84 \%$ did not know cement factory policies, only $33 \%$ of workers had knowledge on all types of PPE measures and $39 \%$ of workers suffered from occupational illness ${ }^{(15)}$. Similarly, Udaypur cement factory workers reflected the high prevalence of respiratory problems. Health problems among workers included musculoskeletal pain $(55.6 \%)$ followed by eye problems (33.2\%). Similarly, $24.1 \%$ had at least one respiratory problem; chest pain and wheezing. Respiratory symptoms were found to be positively associated with low education, duration of work and smoking (16).

Though there exists some research on occupational health in other settings but only few studies on occupational health in the cement factory came in attention in Nepal. This study hopes to raise and strengthen the discussion on worker's health and safety. 


\section{MATERIALS AND METHODS Study design and settings}

A cross-sectional study was used to assess the occupational health risk among cement factory workers of Dang district within the duration of six months; $15^{\text {th }}$ July, 2019 to $15^{\text {th }}$ January 2020. The individual workers working in Samrat cement company private limited, Lamahi and Sonapur minerals and oil private limited, Tulsipur were the study unit.

\section{Sample and sampling}

Proportionate stratified random sampling method was chosen. Stratification was done based on the location of factories registered. Study sample was determined by Cochran formula with prevalence of 39\% percent occupational health risk in Maruti cement factory ${ }^{(15)}$. Total sample of 212 was considered including $10 \%$ of non-response. Staff detail was obtained from the respected factory and random sampling was done using employee serial number and excel random selection procedure. All participants participated in the study. One observation per cement factory was conducted to assess workplace environment.

\section{Data collection}

A structured interview schedule in Nepali and Hindi language was used for face to face interview with factory workers. Also, observation of the cement factory environment was carried out by the observation checklist. No clinical tools were used for disease verification. Only the reported symptoms were considered for the disease. Workers with prior respiratory disease were also included in the study. The occupational health risk tool developed by Syed Sana and et.al $2013^{(17)}$ was used as a reference. Similarly, for knowledge and practice questions, reference of a tool developed by Jay Prakash Sah and et.al $2015^{(15)}$ was used. For the development of the workplace environment checklist, reference to workplace safety and prevention services ${ }^{(18)}$ was used.
The interview schedule was backtranslated (English-Nepali-English/ EnglishHindi-English) and modification was made accordingly as per the context. Pre-testing of the tools was carried out in Manakamana brick industry of Ghorahi, Dang in $10 \%$ of sample size and the improvement in tools was made accordingly.

\section{Operational Definition}

Under occupational health risk, the respiratory and musculoskeletal health problems were considered. Workers with at least anyone symptoms of the respiratory problem and musculoskeletal problem were considered having respiratory health risk and musculoskeletal health risk respectively. Knowledge and practice related questions were asked to participants and the relevant response was scored with 1 and irrelevant response with 0 . Total knowledge score and practice score was converted to percentage and Bloom's cut off point (60 to 80 percent) was used to categorize knowledge into good, fair and poor $^{(19)}$. The average value of the maximum possible score was taken as a cut off value for differentiating workplaces safe and risky.

\section{Data procession and analysis}

Statistical Package for the Social Sciences (SPSS) statistics software version 16 was applied for descriptive and inferential statistical analyses. Only descriptive analysis was conducted regarding workplace status. Univariate and multiple logistic regression models were used to determine the significance and the strength of association to identify the most important independent factors associated with occupational health risks (Yes Vs No). Those variables which showed association during univariate logistic regression were considered for multiple logistic regression.

The odds ratio (OR) and 95\% confidence interval $(\mathrm{CI})$ were reported while showing the association between outcome and independent variables. These results were considered significant at a 5 percent 
Pawan Pandeya et.al. Occupational health risk among selected cement factory workers in dang district of Nepal.

level i.e. p-value $(<0.05)$. During data coding in SPSS for analysis, outcome variable of interest i.e. occupational health risk was coded as 1 and no occupational health risk was coded as 0 . Similarly, for independent variables, the reference category was coded as 0 and the comparing category was coded as 1 .

\section{Ethics statement}

Approval for this study was obtained from the institutional review committee of Manmohan Memorial Institute of Health Sciences with registration number MMIHSIRC 446. Similarly, approval for interviews was obtained from the concerned factory's administration so that workers might feel free to express their views. Informed and written consent was taken before the interview. Participants were ensured of the confidentiality of their responses and were not lured to participate in the study. Workers' queries in the research area were clarified after interview.

\section{RESULTS}

Result part explains the collective findings of both factories. Factory wise characteristics have been displayed in respective tables.

Table 1: Socio-demographic characteristics

\begin{tabular}{|c|c|c|c|}
\hline Socio-demographic characteristics & $\begin{array}{l}\text { Both Factories }(\mathbf{n}=212) \\
\text { Number(Percentage) }\end{array}$ & $\begin{array}{l}\text { Samrat Factory }(n=72) \\
\text { Number(Percentage) }\end{array}$ & $\begin{array}{l}\text { Sonapur Factory }(n=140) \\
\text { Number(Percentage) }\end{array}$ \\
\hline $\begin{array}{l}\text { Age } \\
<=40 \\
>40 \\
\text { Mean } \pm \text { SD;Min-Max }\end{array}$ & $\begin{array}{l}157(74.1) \\
55(25.9) \\
32.6 \pm 10.3 ; 16-64\end{array}$ & $\begin{array}{l}54(75) \\
18(25) \\
32.7 \pm 10.7 ; 16-64\end{array}$ & $\begin{array}{l}103(73.6) \\
37(26.4) \\
32.6 \pm 10.2 ; 17-61\end{array}$ \\
\hline $\begin{array}{l}\text { Gender } \\
\text { Male } \\
\text { Female }\end{array}$ & $\begin{array}{l}196(92.5) \\
16(7.5)\end{array}$ & $\begin{array}{l}71(98.6) \\
1(1.4)\end{array}$ & $\begin{array}{l}125(89.3) \\
15(10.7)\end{array}$ \\
\hline $\begin{array}{l}\text { Marital status } \\
\text { Married } \\
\text { Unmarried } \\
\end{array}$ & $\begin{array}{l}156(73.6) \\
56(26.4)\end{array}$ & $\begin{array}{l}53(73.6) \\
19(26.4) \\
\end{array}$ & $\begin{array}{l}103(73.6) \\
37(26.4)\end{array}$ \\
\hline $\begin{array}{l}\text { Ethnicity } \\
\text { Upper caste group } \\
\text { Disadvantaged janajatis } \\
\text { Dalit } \\
\text { Disadvantaged non-Dalit Terai } \\
\text { Indian people }\end{array}$ & $\begin{array}{l}76(35.8) \\
56(26.4) \\
7(3.3) \\
6(2.8) \\
67(31.6) \\
\end{array}$ & $\begin{array}{l}21(29.2) \\
14(19.4) \\
2(2.8) \\
3(4.2) \\
32(44.4)\end{array}$ & $\begin{array}{l}55(39.3) \\
42(30) \\
5(3.6) \\
3(2.1) \\
35(25) \\
\end{array}$ \\
\hline $\begin{array}{l}\text { Religion } \\
\text { Hindu } \\
\text { Muslim } \\
\text { Buddhist } \\
\end{array}$ & $\begin{array}{l}200(94.3) \\
10(4.7) \\
2(0.9) \\
\end{array}$ & $\begin{array}{l}62(86.1) \\
9(12.5) \\
1(1.4) \\
\end{array}$ & $\begin{array}{l}138(98.6) \\
1(0.7) \\
1(0.7) \\
\end{array}$ \\
\hline $\begin{array}{l}\text { Permanent residence } \\
\text { Province } 1 \\
\text { Province } 2 \\
\text { Bagmati province } \\
\text { Province } 5 \\
\text { Karnali province } \\
\text { Sudurpashchim province } \\
\text { India }\end{array}$ & $\begin{array}{l}6(2.8) \\
12(5.7) \\
2(0.9) \\
102(48.1) \\
3(1.4) \\
21(9.9) \\
67(31.6) \\
\end{array}$ & $\begin{array}{l}1(1.4) \\
2(2.8) \\
1(1.4) \\
31(43.1) \\
1(1.4) \\
4(5.6) \\
32(44.4)\end{array}$ & $\begin{array}{l}5(3.6) \\
10(7.1) \\
0 \\
71(50.7) \\
2(1.4) \\
17(12.1) \\
35(25) \\
\end{array}$ \\
\hline $\begin{array}{l}\text { Education } \\
\text { No formal education } \\
\text { Basic education } \\
\text { Secondary education } \\
\text { Higher education }\end{array}$ & $\begin{array}{l}15(7.1) \\
61(28.8) \\
103(48.6) \\
33(15.6)\end{array}$ & $\begin{array}{l}6(8.3) \\
17(23.6) \\
38(52.8) \\
11(15.3)\end{array}$ & $\begin{array}{l}9(6.4) \\
44(31.4) \\
65(46.4) \\
22(15.7)\end{array}$ \\
\hline $\begin{array}{l}\text { Yearly household income } \\
\text { Enough for }<6 \text { months } \\
\text { Enough for } 6 \text { to } 12 \text { months } \\
\text { Enough for } \geq 12 \text { months }\end{array}$ & $\begin{array}{l}31(14.6) \\
145(68.4) \\
36(17)\end{array}$ & $\begin{array}{l}13(18.1) \\
35(48.6) \\
24(33.3)\end{array}$ & $\begin{array}{l}18(12.9) \\
110(78.6) \\
12(8.6)\end{array}$ \\
\hline
\end{tabular}

Table 1 illustrates the sociodemographic characteristics of factory worker. Study showed the mean age of 212 factory workers as $32.67 \pm 10.35$ years ranging from 16 to 64 years where almost three fourth $(74.1 \%)$ of the workers were $\leq$ to 40 years. The majority $(92.5 \%)$ were male and $73.6 \%$ were married. A high number of upper caste ethnicity $(35.8 \%)$ and the majority $(94.3 \%)$ were Hindu. Almost half $(48.1 \%)$ of the workers were from province 5 while $31.6 \%$ were from India. Only 7.1 percent had no formal education. 
Pawan Pandeya et.al. Occupational health risk among selected cement factory workers in dang district of Nepal.

More than two third had household income enough for 6 to 12 months.

Table 2: Work nature related characteristics

\begin{tabular}{|c|c|c|c|}
\hline Socio-demographic characteristics & $\begin{array}{l}\text { Both Factory }(\mathbf{n}=212) \\
\text { Number(Percentage) }\end{array}$ & $\begin{array}{l}\text { Samrat Factory(n=72) } \\
\text { Number(Percentage) }\end{array}$ & $\begin{array}{l}\text { Sonapur Factory }(\mathbf{n}=140) \\
\text { Number(Percentage) }\end{array}$ \\
\hline $\begin{array}{l}\text { Working industry } \\
\text { Samrat cement industry } \\
\text { Sonapur cement industry }\end{array}$ & $\begin{array}{l}72(34) \\
140(66)\end{array}$ & $\begin{array}{l}72(100) \\
0\end{array}$ & $\begin{array}{l}140(100) \\
0\end{array}$ \\
\hline $\begin{array}{l}\text { Working department } \\
\text { Maintenance } \\
\text { Cement mill } \\
\text { Administration } \\
\text { Security } \\
\text { Transport } \\
\text { Finance/ sales } \\
\text { Production and quality control } \\
\text { Environment } \\
\text { Electrical } \\
\text { Loading/ unloading } \\
\text { Engineering } \\
\text { Health } \\
\end{array}$ & $\begin{array}{l}48(22.6) \\
34(16) \\
25(11.8) \\
21(9.9) \\
17(8) \\
16(7.5) \\
13(6.1) \\
10(4.7) \\
10(4.7) \\
9(4.2) \\
8(3.8) \\
1(0.5) \\
\end{array}$ & $\begin{array}{l}22(30.6) \\
8(11.1) \\
8(11.1) \\
5(6.9) \\
3(4.2) \\
7(9.7) \\
3(4.2) \\
0 \\
3(4.2) \\
7(9.7) \\
5(6.9) \\
1(1.4) \\
\end{array}$ & $\begin{array}{l}26(18.6) \\
26(18.6) \\
17(12.1) \\
16(11.4) \\
14(10) \\
9(6.4) \\
10(7.1) \\
10(7.1) \\
7(5) \\
2(1.4) \\
3(2.1) \\
0 \\
\end{array}$ \\
\hline $\begin{array}{l}\text { Work tenure } \\
<=5 \text { years } \\
>5 \text { years } \\
\text { Mean } \pm \text { SD;Min-Max }\end{array}$ & $\begin{array}{l}179(84.4) \\
33(15.6) \\
2.7 \pm 2.66 ; 0.01-10\end{array}$ & $\begin{array}{l}65(90.3) \\
7(9.7) \\
1.7 \pm 2.3 ; 0.01-10\end{array}$ & $\begin{array}{l}114(81.4) \\
26(18.6) \\
3.2 \pm 2.7 ; 0.04-10\end{array}$ \\
\hline $\begin{array}{l}\text { Work shift } \\
\text { Day } \\
\text { Night }\end{array}$ & $\begin{array}{l}161(75.9) \\
33(24.1) \\
\end{array}$ & $\begin{array}{l}50(69.4) \\
22(30.6) \\
\end{array}$ & $\begin{array}{l}111(79.3) \\
29(20.7)\end{array}$ \\
\hline $\begin{array}{l}\text { Previous occupation } \\
\text { Farmer } \\
\text { Unemployed } \\
\text { Cement factory } \\
\text { Technician } \\
\text { Business } \\
\text { Driver } \\
\text { Foreign employment } \\
\text { Government service } \\
\text { Security guard } \\
\text { Other factories } \\
\text { Others } \\
\end{array}$ & $\begin{array}{l}50(23.6) \\
33(15.6) \\
31(14.6) \\
24(11.3) \\
13(6.1) \\
13(6.1) \\
13(6.1) \\
12(5.7) \\
10(4.7) \\
5(2.4) \\
8(3.8)\end{array}$ & $\begin{array}{l}9(12.5) \\
4(5.6) \\
17(23.6) \\
13(18.1) \\
3(4.2) \\
5(6.9) \\
7(9.7) \\
6(8.3) \\
2(2.8) \\
2(2.8) \\
4(5.6) \\
\end{array}$ & $\begin{array}{l}41(29.3) \\
29(20.7) \\
14(10) \\
11(7.9) \\
10(7.1) \\
8(5.7) \\
6(4.3) \\
6(4.3) \\
8(5.7) \\
3(2.1) \\
4(2.9) \\
\end{array}$ \\
\hline
\end{tabular}

Others $^{*}:$ Teachers, hotel workers, microfinance and consultant

Table 3: Health risk in the factories

\begin{tabular}{|l|l|l|l|}
\hline Health risk & $\begin{array}{l}\text { Both Factories(n=212) } \\
\text { Number(Percentage) }\end{array}$ & $\begin{array}{l}\text { Samrat Factory(n=72) } \\
\text { Number(Percentage) }\end{array}$ & $\begin{array}{l}\text { Sonapur Factory(n=140) } \\
\text { Number(Percentage) }\end{array}$ \\
\hline Respiratory health risk & $28(13.2)$ & $8(11.1)$ & $20(14.3)$ \\
\hline Reported respiratory symptoms & & & \\
Shortness of breath & $75.4 \%$ & $87.5 \%$ & $48.3 \%$ \\
Asthma & $15.4 \%$ & $12.5 \%$ & $31.1 \%$ \\
Wheezing & $6.2 \%$ & 0 & $13.8 \%$ \\
Chest Injuries & $1.5 \%$ & 0 & $3.4 \%$ \\
Cough thick sputum with blood & $1.5 \%$ & 0 & $3.4 \%$ \\
\hline Musculoskeletal health risk & $47(22.2)$ & $10(13.9)$ & $37(26.4)$ \\
\hline Musculoskeletal problems as per body parts & & & \\
Neck & $31.9 \%$ & 0 & $35.7 \%$ \\
Lower Back & $19.1 \%$ & $40 \%$ & $16.7 \%$ \\
Wrist/ Hand & $14.9 \%$ & 0 & $16.7 \%$ \\
Upper Back & $12.8 \%$ & $20 \%$ & $11.9 \%$ \\
Hip/ Thigh & $10.6 \%$ & $40 \%$ & $7.1 \%$ \\
Elbow & $6.4 \%$ & 0 & $7.1 \%$ \\
Shoulder & $4.3 \%$ & 0 & $4.8 \%$ \\
\hline Reported musculoskeletal health problems & & & \\
Difficult to bow down & $30 \%$ & $31.2 \%$ & $29.5 \%$ \\
Tired body & $15 \%$ & $25 \%$ & $11.4 \%$ \\
Heavy head & $10 \%$ & $6.2 \%$ & $11.4 \%$ \\
Staggered in standing up & $10 \%$ & $12.5 \%$ & $9.1 \%$ \\
Stiff/ awkward while moving & $8.3 \%$ & $6.2 \%$ & $9.1 \%$ \\
Heavy feet & $8.3 \%$ & $12.5 \%$ & $6.8 \%$ \\
Sleepy & $5 \%$ & 0 & $6.8 \%$ \\
Thirsty & $5 \%$ & 0 & $6.8 \%$ \\
Heavy eye & $3.3 \%$ & $6.2 \%$ & $2.3 \%$ \\
Yawning & $3.3 \%$ & 0 & $4.5 \%$ \\
Tired talking & $1.7 \%$ & 0 & $2.3 \%$ \\
\hline & & & \\
\hline
\end{tabular}



Nepal.

Work nature related characteristics of factory worker are explained in table 2. Study comprises two third $(66 \%)$ workers from Sonapur cement industry while remaining from Samrat cement industry. Most of the workers were from maintenance department $(22.6 \%)$ followed by a cement mill (16\%), administration (11.8\%), and others as displayed in table. More than three fourth (75.9) had day shift work and $84.4 \%$ had work tenure less than or equal to 5 years. The average duration of work tenure was 2.7 years with maximum tenure of 10 years. Most of the workers had farming $(23.6 \%)$ as the previous occupation followed by a cement factory worker $(14.6 \%)$.

Only $13.2 \%$ of the factory workers reported respiratory risk. Among workers with respiratory health risk, shortness of breath $(75.4 \%)$ was reported by large number of workers. Musculoskeletal health risk was reported by $22.2 \%$. Musculoskeletal problems of neck (31.9\%) was reported by highest number of workers while problem in different body parts included lower back (19.1\%), wrist/ hand
(14.9\%), upper back (12.8\%), hip/ thigh (10.6\%), elbow (6.4 percent) and shoulder (4.3 percent). Comparatively, occupational health risk was reported high in Sonapur cement factory. Details on health risk are shown in table 3.

Health and sanitation related characteristics are portrayed in table 4 in detail. Among total factory workers, $29.2 \%$ had smoking habit. Nearly half of the workers $(48.1 \%)$ used to check their health status half-yearly. Almost all workers (95.8\%) had the availability of first aid services. Similarly, health insurance was available to $64.2 \%$ of workers. More than $95 \%$ of workers reported that the cement factory had installed pollution protective devices.

Hygiene and sanitation indicators were found to be good. Hand washing with soap and water, bathing and changing clothes after work was practiced by $92.5 \%$, $84.4 \%$, and $74.1 \%$ of workers' respectively. More than half $(61.8 \%)$ of the respondents told the floor to be cemented followed by muddy (29.2\%), leveled (8 percent) and graveled (0.9 percent).

Table 4: Health and sanitation related characteristics

\begin{tabular}{|l|l|l|l|}
\hline Health and sanitation related characteristics & $\begin{array}{l}\text { Both Factories(n=212) } \\
\text { Number(Percentage) }\end{array}$ & $\begin{array}{l}\text { Samrat Factory(n=72) } \\
\text { Number(Percentage) }\end{array}$ & $\begin{array}{l}\text { Sonapur Factory(n=140) } \\
\text { Number(Percentage) }\end{array}$ \\
\hline Smoking habit & $62(29.2)$ & $15(20.3)$ & $47(33.6)$ \\
\hline Health service seeking behavior & & 0 & \\
Monthly & $9(4.2)$ & $24(33.3)$ & $9(6.4)$ \\
Half-yearly & $102(48.1)$ & $48(66.7)$ & $78(55.7)$ \\
Yearly & $101(47.6)$ & $72(100)$ & $53(37.9)$ \\
\hline First aid within industry & $203(95.8)$ & $57(79.2)$ & $131(93.6)$ \\
\hline Insurance within industry & $136(64.2)$ & $72(100)$ & $79(56.4)$ \\
\hline Pollution protective device in industry & $202(95.3)$ & $70(97.2)$ & $130(92.9)$ \\
\hline Hand washing with soap \& water & $196(92.5)$ & $56(77.8)$ & $126(90)$ \\
\hline Bath daily after work & $179(84.4)$ & $46(63.9)$ & $123(87.9)$ \\
\hline Change cloth daily after work & $157(74.1)$ & & $111(79.3)$ \\
\hline Floor of industry & & $48(66.7)$ & \\
Cemented & $131(61.8)$ & $21(29.2)$ & $83(59.3)$ \\
Muddy & $62(29.2)$ & $1(1.4)$ & $41(29.3)$ \\
Leveled & $17(8)$ & $2(2.8)$ & $16(11.4)$ \\
Graveled & $2(0.9)$ & & 0 \\
\hline
\end{tabular}

Knowledge related information illustrated in table 5 shows one-third of the respondents $(66 \%)$ had poor knowledge of OHS related questions. Respiratory disease was the highest reported disease $(98.1 \%)$ caused by cement factory while $42 \%$ reported musculoskeletal disease. All the factory workers knew about the mask. More than $80 \%$ of the workers knew the advantages of safety measures where disease prevention was reported by $86.3 \%$ and accident prevention by $83.5 \%$. More than two-thirds $(69.8 \%)$ of the workers knew insurance facilities within the factory. The majority $(90.1 \%)$ had knowledge that compensation is provided for death while 
Pawan Pandeya et.al. Occupational health risk among selected cement factory workers in dang district of Nepal.

$45.8 \%$ knew about the compensation provided for disability.

Table 5: Knowledge related information

\begin{tabular}{|c|c|c|c|}
\hline Knowledge related characteristics & $\begin{array}{l}\text { Both } \\
\text { Factories(n=212) } \\
\text { Number(Percentage) }\end{array}$ & $\begin{array}{l}\text { Samrat } \\
\text { Factory(n=72) } \\
\text { Number(Percentage) }\end{array}$ & $\begin{array}{l}\text { Sonapur } \\
\text { Factory }(n=140) \\
\text { Number(Percentage) }\end{array}$ \\
\hline $\begin{array}{l}\text { Overall knowledge level (Blooms cut off) } \\
\text { Good knowledge } \\
\text { Fair knowledge } \\
\text { Poor knowledge }\end{array}$ & $\begin{array}{l}4(1.9) \\
68(32.1) \\
140(66)\end{array}$ & $\begin{array}{l}0 \\
18(25) \\
54(75)\end{array}$ & $\begin{array}{l}4(2.9) \\
50(35.7) \\
86(61.4)\end{array}$ \\
\hline $\begin{array}{llll}\text { Disease cement industry can cause } & \text { (Multiple } \\
\text { responses) } & & & \\
\text { Respiratory disease } & & & \\
\text { Irritation/ allergy } & & & \\
\text { Musculoskeletal disease } & & & \\
\text { High blood pressure } & & & \\
\text { Swelling of leg/ feet } & & & \\
\text { Heart attack/ failure } & & & \\
\text { Gastrointestinal disease } & & \end{array}$ & $\begin{array}{l}208(98.1) \\
92(43.4) \\
89(42) \\
84(39.6) \\
40(18.9) \\
19(9) \\
15(7.1)\end{array}$ & $\begin{array}{l}72(100) \\
38(52.8) \\
32(44.4) \\
28(38.9) \\
9(12.5) \\
4(5.6) \\
2(2.8)\end{array}$ & $\begin{array}{l}136(97.1) \\
54(38.6) \\
57(40.7) \\
56(40) \\
31(22.1) \\
15(10.7) \\
13(9.3)\end{array}$ \\
\hline $\begin{array}{l}\text { Knowledge on PPE (Multiple responses) } \\
\text { Mask } \\
\text { Boot } \\
\text { Gloves } \\
\text { Goggles } \\
\text { Apron } \\
\text { Helmet } \\
\text { Belt }\end{array}$ & $\begin{array}{l}212(100) \\
184(86.8) \\
159(75) \\
131(61.8) \\
48(22.6) \\
15(7.1) \\
2(0.9) \\
\end{array}$ & $\begin{array}{l}72(100) \\
64(88.9) \\
54(75) \\
47(65.3) \\
12(16.7) \\
11(15.3) \\
72(100) \\
\end{array}$ & $\begin{array}{l}140(100) \\
120(85.7) \\
105(75) \\
84(60) \\
36(25.7) \\
4(2.9) \\
2(1.4) \\
\end{array}$ \\
\hline $\begin{array}{l}\text { Advantage of safety measures (Multiple responses) } \\
\text { Disease prevention } \\
\text { Accident prevention }\end{array}$ & $\begin{array}{l}183(86.3) \\
177(83.5)\end{array}$ & $\begin{array}{l}71(98.6) \\
49(68.1)\end{array}$ & $\begin{array}{l}112(80) \\
128(91.4)\end{array}$ \\
\hline Knowledge of insurance facility & $148(69.8)$ & $57(79.2)$ & $91(65)$ \\
\hline $\begin{array}{l}\text { Knowledge of policies within the industry (Multiple } \\
\text { responses) } \\
\text { Health insurance related policy } \\
\text { Salary related policy } \\
\text { Leave related policy } \\
\text { OHS related policy } \\
\text { Work hour related policy } \\
\text { Social security related policy }\end{array}$ & $\begin{array}{l}109(51.4) \\
95(44.8) \\
92(43.4) \\
68(32.1) \\
45(21.2) \\
28(13.2)\end{array}$ & $\begin{array}{l}42(58.3) \\
14(19.4) \\
16(22.2) \\
20(27.8) \\
10(13.9) \\
5(6.9)\end{array}$ & $\begin{array}{l}67(47.9) \\
81(57.9) \\
76(54.3) \\
48(34.3) \\
35(25) \\
23(16.4)\end{array}$ \\
\hline $\begin{array}{l}\text { Knowledge of compensation (Multiple responses) } \\
\text { For death } \\
\text { For disability }\end{array}$ & $\begin{array}{l}\text { 191(90.1) } \\
97(45.8)\end{array}$ & $\begin{array}{l}64(88.9) \\
41(56.9)\end{array}$ & $\begin{array}{l}127(90.7) \\
56(40)\end{array}$ \\
\hline
\end{tabular}

Table 6 explains on source of safety information where more than three quarter $(75.9 \%)$ got information through peers followed by television (61.3\%), training/ orientation $(59.9 \%)$, radio $(55.2 \%)$ and newspaper $(21.7 \%)$. More than one fourth
(29.7\%) had not received training on OHS. Among training receiver $(70.3 \%)$, majority $(93.2 \%)$ got training on safety measures while 6.71 percent got training on near-miss accident/ hazard control.

Table 6: Training and information source related knowledge

\begin{tabular}{|l|l|l|l|}
\hline Training and information source related knowledge & $\begin{array}{l}\text { Both Factories(n=212) } \\
\text { Number(Percentage) }\end{array}$ & $\begin{array}{l}\text { Samrat Factory(n=72) } \\
\text { Number(Percentage) }\end{array}$ & $\begin{array}{l}\text { Sonapur Factory(n=140) } \\
\text { Number(Percentage) }\end{array}$ \\
\hline Source of safety information (Multiple responses) & & & \\
Peers & $161(75.9)$ & $56(77.8)$ & $105(75)$ \\
Television & $130(61.3)$ & $18(25)$ & $112(80)$ \\
Training/ orientation & $127(59.9)$ & $57(79.2)$ & $70(50)$ \\
Radio & $117(55.2)$ & $10(13.9)$ & $107(76.4)$ \\
Newspaper & $46(21.7)$ & $10(13.9)$ & $36(25.7)$ \\
\hline Received training on OHS & $149(70.3)$ & $57(79.2)$ & $92(65.7)$ \\
\hline Training topic & $(\mathrm{n}=149)$ & $(\mathrm{n}=57)$ & $(\mathrm{n}=92)$ \\
Safety measures training & $139(93.2)$ & $56(98.2)$ & $83(90.2)$ \\
Near miss accident/ hazard control & $10(6.71)$ & $1(1.8)$ & $9(9.8)$ \\
\hline
\end{tabular}

Table 7 shows only $17.9 \%$ had poor practice to prevent respiratory problems while 41 percent had poor practice to prevent musculoskeletal problems. The majority (87.7\%) used PPE. Among PPE users, mask users $(85.8 \%)$ were in high 
Pawan Pandeya et.al. Occupational health risk among selected cement factory workers in dang district of Nepal.

numbers to prevent different health risk. Nearly three fourth $(73.1 \%)$ used all required PPE. Among those who don't use
PPE, most of the workers $(47.3 \%)$ replied no need followed by discomfort $(43.8 \%)$ and not available ( 8.7 percent).

Table 7: Practice related information

\begin{tabular}{|c|c|c|c|}
\hline Practice related characteristics & $\begin{array}{l}\text { Both Factories }(\mathrm{n}=\mathbf{2 1 2}) \\
\text { Number(Percentage) }\end{array}$ & $\begin{array}{l}\text { Samrat Factory }(n=72) \\
\text { Number(Percentage) }\end{array}$ & $\begin{array}{l}\text { Sonapur Factory }(n=140) \\
\text { Number(Percentage) }\end{array}$ \\
\hline $\begin{array}{lll}\text { Practice to prevent } & \text { respiratory } & \text { problem } \\
\text { (Blooms cut off) } & & \\
\text { Good practice } & & \\
\text { Fair practice } & & \\
\text { Poor practice } & & \\
\end{array}$ & $\begin{array}{l}59(27.8) \\
115(54.2) \\
38(17.9)\end{array}$ & $\begin{array}{l}20(27.8) \\
44(61.1) \\
8(11.1)\end{array}$ & $\begin{array}{l}39(27.9) \\
71(50.7) \\
30(21.4)\end{array}$ \\
\hline $\begin{array}{l}\text { Practice to prevent MSD problem (Blooms cut } \\
\text { off) } \\
\text { Good practice } \\
\text { Fair practice } \\
\text { Poor practice }\end{array}$ & $\begin{array}{l}42(19.8) \\
83(39.2) \\
87(41)\end{array}$ & $\begin{array}{l}15(20.8) \\
36(50) \\
21(29.2)\end{array}$ & $\begin{array}{l}27(19.3) \\
47(33.6) \\
66(47.1)\end{array}$ \\
\hline PPE use & $186(87.7)$ & $71(98.6)$ & $115(82.1)$ \\
\hline $\begin{array}{l}\text { PPE use by types (Multiple responses) } \\
\text { Mask } \\
\text { Boot } \\
\text { Gloves } \\
\text { Goggles } \\
\text { Helmet } \\
\text { Apron } \\
\text { Belt } \\
\end{array}$ & $\begin{array}{l}182(85.8) \\
129(60.8) \\
92(43.4) \\
75(35.4) \\
41(19.3) \\
24(11.3) \\
3(1.4)\end{array}$ & $\begin{array}{l}69(95.8) \\
52(72.2) \\
26(36.1) \\
28(38.9) \\
27(37.5) \\
9(12.5) \\
1(1.4)\end{array}$ & $\begin{array}{l}113(80.7) \\
77(55) \\
66(47.1) \\
47(33.6) \\
14(10) \\
15(10.7) \\
2(1.4)\end{array}$ \\
\hline $\begin{array}{l}\text { Use of all required PPE } \\
\text { Reason for not using all required PPE } \\
\text { Not necessary } \\
\text { Discomfort } \\
\text { Not available }\end{array}$ & $\begin{array}{l}155(73.1) \\
(\mathrm{n}=57) \\
27(47.3) \\
25(43.8) \\
5(8.7)\end{array}$ & $\begin{array}{l}54(75) \\
(\mathrm{n}=18) \\
14(77.8) \\
3(16.7) \\
1(5.5)\end{array}$ & $\begin{array}{l}101(72.1) \\
(\mathrm{n}=39) \\
13(33.3) \\
22(56.4) \\
4(10.3)\end{array}$ \\
\hline
\end{tabular}

Table 8: Enabling and reinforcing factors for the practice of safety measures

\begin{tabular}{|c|c|c|c|}
\hline Practice related characteristics & $\begin{array}{l}\text { Both Factories(n=212) } \\
\text { Number(Percentage) }\end{array}$ & $\begin{array}{l}\text { Samrat Factory }(n=72) \\
\text { Number(Percentage) }\end{array}$ & $\begin{array}{l}\text { Sonapur Factory }(n=140) \\
\text { Number(Percentage })\end{array}$ \\
\hline $\begin{array}{l}\text { Accessibility of PPE } \\
\text { If accessible, the timing of availability } \\
\text { As per requirement } \\
\text { Half-yearly } \\
\text { Yearly }\end{array}$ & $\begin{array}{l}204(96.2) \\
(\mathrm{n}=204) \\
174(85.3) \\
15(7.3) \\
15(7.3)\end{array}$ & $\begin{array}{l}72(100) \\
(\mathrm{n}=72) \\
72(100) \\
0 \\
0\end{array}$ & $\begin{array}{l}132(94.3) \\
(\mathrm{n}=132) \\
102(77.2) \\
15(11.4) \\
15(11.4)\end{array}$ \\
\hline $\begin{array}{l}\text { Condition of PPE } \\
\text { Good } \\
\text { Poor }\end{array}$ & $\begin{array}{l}194(91.5) \\
18(8.5)\end{array}$ & $\begin{array}{l}70(97.2) \\
2(2.8)\end{array}$ & $\begin{array}{l}124(88.6) \\
16(11.4)\end{array}$ \\
\hline Compulsion on safety measures used in industry & $180(84.9)$ & $71(98.6)$ & 109(77.9) \\
\hline $\begin{array}{l}\text { Monitoring of safety measures use in industry } \\
\text { If yes, safety measures use monitoring schedule } \\
\text { Daily } \\
\text { Weekly } \\
\text { Monthly }\end{array}$ & $\begin{array}{l}184(86.8) \\
(\mathrm{n}=184) \\
172(93.4) \\
9(4.8) \\
3(1.6)\end{array}$ & $\begin{array}{l}69(95.8) \\
(\mathrm{n}=69) \\
67(97.1)) \\
2(2.9)) \\
0\end{array}$ & $\begin{array}{l}115(82.1) \\
(\mathrm{n}=115) \\
105(91.3) \\
7(6.1) \\
3(2.6)\end{array}$ \\
\hline $\begin{array}{l}\text { Penalty for no PPE use } \\
\text { Type of penalty } \\
\text { Warning } \\
\text { Absent/ fine } \\
\text { Counselling/ orientation }\end{array}$ & $\begin{array}{c}\begin{array}{c}137(64.6) \\
(\mathrm{n}=137)\end{array} \\
63(45.9) \\
55(40.1) \\
19(13.8)\end{array}$ & $\begin{array}{l}\begin{array}{l}64(88.9) \\
(n=64)\end{array} \\
24(37.5) \\
36(56.3) \\
4(6.2)\end{array}$ & 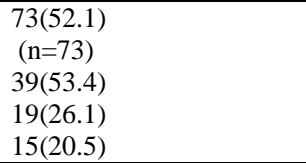 \\
\hline Monitoring from the government side for safety use & $106(50)$ & $44(61.1)$ & $62(44.3)$ \\
\hline
\end{tabular}

Enabling and reinforcing factors for the practice of safety measures is displayed in table 8. Of total factory workers, only 3.8 percent had no access to PPE. Among those who had access to PPE, $85.3 \%$ had access as per requirement while 7.3 percent had access in half yearly and yearly. Majority $(91.5 \%)$ of the workers had PPE in good condition. The industry had made compulsion on the use of safety measures which was reported by $84.9 \%$ of workers. Monitoring of safety measures used in industry from factory administration was reported by $86.8 \%$ where $93.4 \%$ reported daily monitoring while 4.8 percent and 1.6 percent reported weekly and monthly monitoring respectively. Almost two-third $(64.6 \%)$ replied that there is a provision of penalty for no use of safety measures. Warning (45.9\%), absent/ fine $(40.1 \%)$ and 
Pawan Pandeya et.al. Occupational health risk among selected cement factory workers in dang district of Nepal.

counselling/ orientation (13.8\%) were the penalty applied for no use of safety measures. More than $40 \%$ told that, the penalty in the form of absent or fine. Half of the factory workers replied the practice of monitoring from government side for safety.

Association of occupational health risk with different variables is explained in table 9 where none of the sociodemographic variables showed a statistically significant association with respiratory health risk while residence $(\mathrm{p}=0.018$, $\mathrm{COR}=0.37,95 \%$ CI: 0.16-0.84), educational status ( $\mathrm{p}=0.024, \mathrm{COR}=3.43,95 \% \mathrm{CI}: 1.18$ 10.03) and work shift ( $\mathrm{p}=0.011, \mathrm{COR}=2.49$, 95\% CI: 1.23-5.01) had statistically significant association with musculoskeletal health risk. Workers age more than 40 years had 2.05 times high risk of respiratory risk while 1.89 times high risk of musculoskeletal risk.

Table 9: Association between health risk and socio demographic characteristics

\begin{tabular}{|c|c|c|c|c|c|c|}
\hline \multirow{2}{*}{ Socio demographic characteristics } & \multicolumn{3}{|c|}{ Respiratory health risk } & \multicolumn{3}{|c|}{ Musculoskeletal health risk } \\
\hline & p-value & COR & $95 \% \mathrm{CI}$ & p-value & COR & $95 \% \mathrm{CI}$ \\
\hline $\begin{array}{l}\text { Age } \\
>40 \\
<=40\end{array}$ & 0.088 & $\begin{array}{l}2.05 \\
\text { Ref }\end{array}$ & $0.21-1.11$ & 0.072 & $\begin{array}{l}1.89 \\
\text { Ref }\end{array}$ & $0.94-3.80$ \\
\hline $\begin{array}{l}\text { Gender } \\
\text { Male } \\
\text { Female }\end{array}$ & 0.407 & $\begin{array}{l}2.39 \\
\text { Ref }\end{array}$ & $0.30-18.88$ & 0.777 & $\begin{array}{l}0.84 \\
\text { Ref }\end{array}$ & $0.26-2.75$ \\
\hline $\begin{array}{l}\text { Marital status } \\
\text { Married } \\
\text { Unmarried } \\
\end{array}$ & 0.522 & $\begin{array}{l}1.36 \\
\text { Ref }\end{array}$ & $0.52-3.57$ & 0.204 & $\begin{array}{l}\text { Ref } \\
0.59\end{array}$ & $0.27-1.33$ \\
\hline $\begin{array}{l}\text { Ethnic group } \\
\text { Other castes } \\
\text { Upper caste }\end{array}$ & 0.661 & $\begin{array}{l}1.20 \\
\text { Ref }\end{array}$ & $0.51-2.82$ & 0.958 & $\begin{array}{l}0.98 \\
\text { Ref }\end{array}$ & $0.50-1.93$ \\
\hline $\begin{array}{l}\text { Religion } \\
\text { Other } \\
\text { Hindu }\end{array}$ & 0.716 & $\begin{array}{l}1.33 \\
\text { Ref }\end{array}$ & $0.27-6.45$ & 0.810 & $\begin{array}{l}1.18 \\
\text { Ref }\end{array}$ & $0.31-4.55$ \\
\hline $\begin{array}{l}\text { Permanent Residence } \\
\text { Native } \\
\text { Non-native }\end{array}$ & 0.214 & $\begin{array}{l}\text { Ref } \\
0.55\end{array}$ & $0.21-1.42$ & 0.018 & $\begin{array}{l}\text { Ref } \\
0.37\end{array}$ & $0.16-0.84$ \\
\hline $\begin{array}{l}\text { Educational status } \\
\text { No formal education } \\
\text { Formal education }\end{array}$ & 0.449 & $\begin{array}{l}0.45 \\
\text { Ref }\end{array}$ & $0.05-3.56$ & 0.024 & $\begin{array}{l}3.43 \\
\text { Ref }\end{array}$ & $1.18-10.03$ \\
\hline $\begin{array}{l}\text { Working nature } \\
\text { Admin/Table Worker } \\
\text { Field level Worker }\end{array}$ & 0.920 & $\begin{array}{l}\text { Ref } \\
1.04\end{array}$ & $0.46-2.32$ & 0.781 & $\begin{array}{l}\text { Ref } \\
1.16\end{array}$ & $0.41-3.27$ \\
\hline $\begin{array}{l}\text { Tenure } \\
>5 \text { years } \\
<=5 \text { years }\end{array}$ & 0.146 & $\begin{array}{l}2.02 \\
\text { Ref }\end{array}$ & $0.78-5.24$ & 0.444 & $\begin{array}{l}1.39 \\
\text { Ref }\end{array}$ & $0.59-3.24$ \\
\hline $\begin{array}{l}\text { Work shift } \\
\text { Night } \\
\text { Day } \\
\end{array}$ & 0.727 & $\begin{array}{l}0.84 \\
\text { Ref }\end{array}$ & $0.32-2.20$ & 0.011 & $\begin{array}{l}2.49 \\
\text { Ref }\end{array}$ & $1.23-5.01$ \\
\hline $\begin{array}{l}\text { Previous occupation } \\
\text { With dust exposure } \\
\text { Without dust exposure }\end{array}$ & 0.535 & $\begin{array}{l}1.28 \\
\text { Ref }\end{array}$ & $0.58-2.85$ & NA & NA & NA \\
\hline $\begin{array}{l}\text { HH yearly income } \\
\text { Enough }<6 \text { months } \\
\text { Enough }>6 \text { months }\end{array}$ & 0.532 & $\begin{array}{l}0.66 \\
\text { Ref }\end{array}$ & $0.18-2.36$ & 0.384 & $\begin{array}{l}0.64 \\
\text { Ref }\end{array}$ & $0.23-1.76$ \\
\hline
\end{tabular}

Table 10 shows statistically significant association of respiratory health risk with smoking habit $(\mathrm{p}=0.003$, $\mathrm{COR}=3.36,95 \% \mathrm{CI}: 1.49-7.58)$ and access to safety measures $(\mathrm{p}=0.006, \mathrm{COR}=0.13$, 95\% CI: 0.03-0.56). Smokers had more than three times the chance of respiratory risk compared to a non-smoker. Similarly, workers with access to safety measures were $87 \%$ protective to respiratory health risk. Musculoskeletal health risk was statistically significantly associated with availability of first aid services $(\mathrm{p}=0.001, \mathrm{COR}=0.07,95 \%$ CI: 0.01-0.35). Workers with access to first aid service were $93 \%$ protective to musculoskeletal health risk. 
Pawan Pandeya et.al. Occupational health risk among selected cement factory workers in dang district of Nepal.

Table 10: Association between health risk and health related characteristics

\begin{tabular}{|c|c|c|c|c|c|c|}
\hline \multirow{2}{*}{ Health related characteristics } & \multicolumn{3}{|c|}{ Respiratory health risk } & \multicolumn{3}{|c|}{ Musculoskeletal health risk } \\
\hline & p-value & COR & $95 \% \mathrm{CI}$ & p-value & COR & $95 \% \mathrm{CI}$ \\
\hline $\begin{array}{l}\text { Smoking habit } \\
\text { Yes } \\
\text { No }\end{array}$ & $0.003^{*}$ & $\begin{array}{l}3.36 \\
\text { Ref }\end{array}$ & $1.49-7.58$ & NA & NA & NA \\
\hline $\begin{array}{l}\text { Health check-up } \\
\text { Within a year } \\
\text { Yearly }\end{array}$ & 0.078 & $\begin{array}{l}2.11 \\
\text { Ref }\end{array}$ & $0.90-4.91$ & 0.429 & $\begin{array}{l}1.30 \\
\text { Ref }\end{array}$ & $0.68-2.50$ \\
\hline $\begin{array}{l}\text { Health service within factory } \\
\text { Available } \\
\text { Not available }\end{array}$ & 0.970 & $\begin{array}{l}1.01 \\
\text { Ref }\end{array}$ & $0.38-2.68$ & 0.130 & $\begin{array}{l}0.57 \\
\text { Ref }\end{array}$ & $0.27-1.18$ \\
\hline $\begin{array}{l}\text { First aid available } \\
\text { Yes } \\
\text { No }\end{array}$ & 0.850 & $\begin{array}{l}1.22 \\
\text { Ref }\end{array}$ & $0.14-10.20$ & 0.001 & $\begin{array}{l}0.07 \\
\text { Ref }\end{array}$ & $0.01-0.35$ \\
\hline $\begin{array}{l}\text { Received insurance } \\
\text { Yes } \\
\text { No }\end{array}$ & 0.094 & $\begin{array}{l}2.25 \\
\text { Ref }\end{array}$ & $0.87-5.82$ & 0.279 & $\begin{array}{l}0.69 \\
\text { Ref }\end{array}$ & $0.36-1.35$ \\
\hline $\begin{array}{l}\text { Received OHS training } \\
\text { Yes } \\
\text { No }\end{array}$ & 0.457 & $\begin{array}{l}0.72 \\
\text { Ref }\end{array}$ & $0.31-1.68$ & 0.147 & $\begin{array}{l}0.60 \\
\text { Ref }\end{array}$ & $0.31-1.19$ \\
\hline $\begin{array}{l}\text { Knowledge on OHS } \\
\text { Good } \\
\text { Poor } \\
\end{array}$ & 0.524 & $\begin{array}{l}1.30 \\
\text { Ref }\end{array}$ & $0.33-1.73$ & 0.303 & $\begin{array}{l}0.69 \\
\text { Ref }\end{array}$ & $0.34-1.40$ \\
\hline $\begin{array}{l}\text { Access to safety measure } \\
\text { Yes } \\
\text { No }\end{array}$ & $0.006^{*}$ & $\begin{array}{l}0.13 \\
\text { Ref }\end{array}$ & $0.03-0.56$ & 0.070 & $\begin{array}{l}0.27 \\
\text { Ref }\end{array}$ & $0.07-1.11$ \\
\hline $\begin{array}{l}\text { Practice of safety measures } \\
\text { Yes } \\
\text { No }\end{array}$ & 0.120 & $\begin{array}{l}0.44 \\
\text { Ref }\end{array}$ & $0.16-1.23$ & 0.108 & $\begin{array}{l}0.49 \\
\text { Ref }\end{array}$ & $0.20-1.18$ \\
\hline $\begin{array}{l}\text { Mask use } \\
\text { Yes } \\
\text { No }\end{array}$ & 0.084 & $\begin{array}{l}0.42 \\
\text { Ref }\end{array}$ & $0.16-1.12$ & NA & NA & NA \\
\hline $\begin{array}{l}\text { Condition of safety measures } \\
\text { Good } \\
\text { Poor }\end{array}$ & 0.066 & $\begin{array}{l}0.35 \\
\text { Ref }\end{array}$ & $0.11-1.07$ & 0.082 & $\begin{array}{l}0.41 \\
\text { Ref }\end{array}$ & $0.15-1.12$ \\
\hline
\end{tabular}

Multiple logistic regression analysis is depicted in table 11. Variables that showed statistically significant relationship in univariate logistic regression were considered for multiple logistic regression. Smoking and access to safety measures showed statistically significant relationship with respiratory health risk. Similarly, residence, educational status and work shift showed statistically significant relationship with musculoskeletal health risk.
The respiratory health risk was found to be associated with smoking $(\mathrm{AOR}=3.40$, $\mathrm{p}=0.004$, 95\% CI: $1.47-7.85)$. Smokers were more than 2 times at risk of respiratory problems compared to non- smokers. Likewise, respiratory risk and access to safety measures were statistically significant (AOR=0.12, $\mathrm{p}=0.008,95 \%$ CI: 0.02-0.58). Those workers who had access to safety measures had $88 \%$ less chance to develop respiratory problems.

Table 11: Multiple logistic regression analysis

\begin{tabular}{|c|c|c|c|c|c|c|c|}
\hline \multirow{2}{*}{ Health related characteristics } & \multicolumn{3}{|c|}{ Respiratory health risk } & \multirow{2}{*}{ Health related characteristics } & \multicolumn{3}{|c|}{ Musculoskeletal health risk } \\
\hline & AOR & p-value & CI & & AOR & p-value & CI \\
\hline $\begin{array}{l}\text { Smoking } \\
\text { No } \\
\text { Yes }\end{array}$ & $\begin{array}{l}\text { Ref } \\
3.40\end{array}$ & $0.004^{*}$ & $1.47-7.85$ & $\begin{array}{l}\text { Permanent Residence } \\
\text { Native } \\
\text { Non-native }\end{array}$ & $\begin{array}{l}\text { Ref } \\
0.44\end{array}$ & 0.061 & $0.19-1.04$ \\
\hline \multirow[t]{3}{*}{$\begin{array}{l}\text { Access to safety measures } \\
\text { No } \\
\text { Yes }\end{array}$} & \multirow{3}{*}{$\begin{array}{l}\text { Ref } \\
0.12\end{array}$} & \multirow{3}{*}{$0.008^{*}$} & \multirow{3}{*}{$0.02-0.58$} & $\begin{array}{l}\text { Educational status } \\
\text { No formal education } \\
\text { Formal education }\end{array}$ & $\begin{array}{l}3.77 \\
\text { Ref }\end{array}$ & 0.021 & $1.22-11.69$ \\
\hline & & & & $\begin{array}{l}\text { Work shift } \\
\text { Night } \\
\text { Day }\end{array}$ & $\begin{array}{l}2.44 \\
\text { Ref }\end{array}$ & 0.022 & $1.14-5.22$ \\
\hline & & & & $\begin{array}{l}\text { First aid available } \\
\text { Yes } \\
\text { No }\end{array}$ & $\begin{array}{l}0.11 \\
\text { Ref }\end{array}$ & 0.009 & $0.02-0.58$ \\
\hline
\end{tabular}


The musculoskeletal health risk was found to be associated with educational status $(\mathrm{AOR}=3.77, \mathrm{p}=0.021,95 \% \mathrm{CI}: 1.22-$ 11.69), work shift (AOR $=2.44, \mathrm{p}=0.022$, 95\% CI: 1.14-5.22) and first aid service availability $(\mathrm{AOR}=0.11, \mathrm{p}=0.009,95 \% \mathrm{CI}$ : 0.02-0.58). Workers with no formal education and day-time work shift were 3.77 and 2.44 times at risk to musculoskeletal health risk. Similarly, workers with access to first aid services were $89 \%$ protective to musculoskeletal health risk.

Table 12 shows both cement factories had safe workplace environment. Of the total 36 workplace environment related indicators, Sonapur cement factory (91.67\%) had more positive indicators compared to the Samrat cement factory $(86.11 \%)$.

Table 12: Factory workplace environment status

\begin{tabular}{|l|l|l|l|}
\hline \multirow{2}{*}{$\begin{array}{l}\text { Cement } \\
\text { factory }\end{array}$} & $\begin{array}{l}\text { Workplace indicator (n=2; } \\
\text { indicators=36) }\end{array}$ & $\begin{array}{l}\text { Workplace } \\
\text { status }\end{array}$ \\
\cline { 2 - 4 } & Positive n(\%) & Negative n(\%) & \\
\hline Samrat & $31(86.11)$ & $5(13.89)$ & Safe \\
\hline Sonapur & $33(91.67)$ & $3(8.33)$ & Safe \\
\hline \multicolumn{4}{|c|}{$n(\%):$ Number(Percentage) }
\end{tabular}

\section{DISCUSSION}

Present study showed $13.2 \%$ of workers have respiratory risk while a similar study conducted in Maruti cement factory, Siraha and Udaypur cement factory showed $39 \%$ and $24.1 \%$ workers respectively were suffering from any illness ${ }^{(15,16)}$. But, finding of the Dejen cement factory showed higher prevalence $(62.9 \%){ }^{(20)}$. This might be due to high PPE use $(87.7 \%)$, strict monitoring regarding use of safety measures $(86.8 \%)$, provision of safety training (70.3\%), etc.

As per the present study, $75.4 \%$ have shortness of breath, $15.4 \%$ have asthma, 6.2 percent have wheezing, 1.5 percent have chest injuries and 1.5 percent have a cough which is lower than the Dejen cement factory as shortness of breath (38.6\%), wheezing $(36.9 \%)$, chest pain $(21 \%)$ and $24.5 \%$ had a cough ${ }^{(20)}$. Study of Udaypur cement factory showed chronic cough was present in 3.4 percent, 9.2 percent had wheezing and asthma in 2 percent of workers ${ }^{(16)}$. Similarly, 54\% had wheezing as respiratory symptoms in the study conducted in Pakistan ${ }^{(23)}$. Comparatively low respiratory symptom was observed in the present study which might be due to high PPE use and installation of pollution control devices.

The current study report MSD among $22.2 \%$ of worker which is consistent with Portland cement factory where only $18.8 \%$ of workers had musculoskeletal health risk ${ }^{(24)}$. MSD might be high in this study since it is neglected as it does not cause serious effect at initial stage and is not taken as a disease. So, most of them might not get treated on time.

In this study MSD was found in neck $(31.9 \%)$, shoulder (4.3 percent), upper back (12.8\%), elbow (6.4 percent), lower back (19.2\%), wrist/ hand (14.9\%), Hip/ thigh (10.6\%). Musculoskeletal problem was observed to be high among cement factory workers as per study carried out by IOS Press and the authors i.e. in neck $(36 \%)$, shoulder $(30 \%)$, upper back $(15 \%)$, elbow (17\%), low back (31\%), Wrist/ hands (17\%), Hips/ thighs $(14 \%)^{(25)}$.

But comparatively less musculoskeletal health risk was observed in the study conducted among workers of construction industries in Chennai metropolitan city. Musculoskeletal health problems included problems in neck (9.7 percent), shoulder (10.4\%), upper back (7.8 percent), elbow (7.8 percent), lower back problems $(20.8 \%)$, wrist problem $(11 \%)$ and hips/ thigh (6.5 percent) ${ }^{(26)}$.

As per this study, 29.2\% worker smoke cigarette while higher number of Udaypur cement factory workers, Qassim cement factory workers a, South Indian cement factory workers and Dejen cement factory workers i.e. $33.2 \%, 46 \%, 59.1 \%$ and $11.6 \%$ currently were smoker respectively $(16,21,22,20)$

This study reflects that $66 \%$ of workers have poor knowledge on occupational health and safety. Similar finding was seen in the study of Anand Sundarar in India which showed $64.6 \%$ had 
poor knowledge ${ }^{(21)}$. In the Maruti cement factory, $98 \%$ had knowledge of the benefits of PPE while in this study $86 \%$ and $83.5 \%$ told disease prevention and accident prevention as benefits of PPE. Similarly, $90 \%$ of workers have received training while only $70.3 \%$ received training on occupational health and safety in the present study ${ }^{(15)}$.

Among total study participants, $87.7 \%$ use PPE in this study while only $79 \%$ used in Udaypur cement factory ${ }^{(16)}$ and $31.2 \%$ used in cement factory of Ethiopia (20), $96 \%$ used in Maruti cement factory ${ }^{(15)}$ and $99 \%$ of the workers used PPE as per the study of Aliae Mohamed Hussain ${ }^{(27)}$. Of the total PPE user in the current study, $85.8 \%$ use mask, $60.8 \%$ use boot, $43.4 \%$ use gloves, $11.3 \%$ use apron, $35.4 \%$ use goggles. Among total PPE users in the Udaypur cement factory, $80.3 \%$ used mask, $30.2 \%$ used boot, $26.8 \%$ used gloves, $18.6 \%$ used apron, and $12.2 \%$ used goggles ${ }^{(16)}$.

In the study conducted by Anand Sundarraj ${ }^{(21)}$, reasons for no use of PPE were not availability (30.8\%), not necessary $(21.9 \%)$ and uncomfortable (47\%) while in this study, the reasons are not availability (8.7 percent), not necessary $(47.3 \%)$ and uncomfortable (43.8\%).

This study reflects that the risk of respiratory diseases is reduced by $28 \%$ among OHS training receiver. A similar study in Northwest Ethiopia reflected that training on occupational health and safetyrelated to dust, health effect reduced respiratory symptoms by $82 \%$ (AOR $=0.18$ 95\% CI: $0.09-0.36){ }^{(11)}$. The association might have been affected because onefourth of the respondent does not use all required safety measures (26.9\%).

Statistically, the significant relationship is obtained between respiratory risk and smoking where smokers have more than three times higher risk compared to non-smokers $(\mathrm{AOR}=3.40, \mathrm{p}=0.004,95 \%$ CI: 1.47-7.85) in this study which is in line to the study of Dejen cement factory where smokers developed respiratory symptoms
5.38 times more likely $(\mathrm{AOR}=5.38,95 \% \mathrm{CI}$ : $1.42,20.39)$ than non-smokers ${ }^{(20)}$.

This study reflects that there is 1.28 times high chance of respiratory risk among workers with previous occupation with dust exposure while in cement factory workers of Northwest Ethiopia, the occupational history of dust exposure was likely to develop respiratory symptoms $(\mathrm{AOR}=2.94$, 95\% CI: $1.13-7.63)^{(11)}$. Workers aged $>40$ years were two times more likely to develop respiratory risk in this study which coincides with a similar finding in the study of Dejel cement factory where $\geq 45$ years were four times more likely to develop respiratory symptoms ${ }^{(20)}$.

Education $(\mathrm{p}=0.449)$, work tenure (0.146), age (0.088), gender (0.407), work shift (0.727), are not found to be significantly associated while smoking is significantly associated with respiratory disease $(p=0.020)$ in this study. Study in Udaypur cement factory showed significant association among respiratory symptoms and education (0.009), duration of work $(<0.001)$, smoking $(<0.001)$ while age $(0.856)$, gender (0.929), work shift (0.19) were not significantly associated ${ }^{(16)}$.

Present study revealed that no statistical association was observed among musculoskeletal health risk and age $(\mathrm{p}=0.072)$, and job tenure $(\mathrm{p}=0.444)$. Study in Iranian Zinc Industry, showed association of MSD and age $(<0.001)$ and job tenure $(<0.001)^{(28)}$. Similarly, the study conducted in India showed workers working more than 11 years had 2.77 times more risk (AOR=2.77, CI: 1.12-6.83) ${ }^{(26)}$. This might be affected due to small sample size where workers working more than 5 years cover only $15.6 \%$ of total sample.

This study shows that workers above 40 years has 1.89 times risk of musculoskeletal problems which is consistent with the study conducted in India where workers aged 41 years and above had 2.72 times more risk of musculoskeletal morbidities (AOR=2.72, CI: 0.99-7.47). Gender had no significant association with MSD where males were 7 percent protective 
compared to females in the study of India which is consistent with the present findings where male were $16 \%$ protective to musculoskeletal problems ${ }^{(26)}$.

In the univariate logistic analysis, some cell frequencies are very low which might have affected the relationship. Though the confidence interval is unidirectional in multiple logistic regression the range is a little bit large. Despite all this information, smoking and access to safety measures are found to be a predictor of respiratory health risk. Similarly, educational status, work shift and first aid availability were found to be the predictors of musculoskeletal health risk.

\section{CONCLUSION}

Occupational health risk was reported less compared to similar study carried out in Nepal although the risk is still high. Musculoskeletal health risk was reported to be high by both cement factory workers. Special protection/ care needs to be provided to workers above 40 years of age since risk increases as per age. Field level workers, with special focus, need to be fully equipped with necessary safety measures to prevent health risk. Cement factories could be declared as the smoking restricted area. Behavior change communication related to OHS could be adopted to ensure workers' compliance with safety devices which improves knowledge level and ultimately healthy practice will be achieved. Routine health examination could help workers identify health issues timely to prevent complications. The government should strictly execute protocol and standards to monitor OHS.

\section{AUTHOR CONTRIBUTIONS}

Research Conceptualization: Pawan

Pandeya, Arzoo Neupane, Durga Khadka Mishra, Shankar Prasad Khanal, Yadav Prasad Joshi, Sujan Babu Marahatta

Data Collection: Pawan Pandeya, Arzoo Neupane
Statistical Analysis: Pawan Pandeya, Shankar Prasad Khanal, Rashmita Chhettri, Arzoo Neupane

Writing manuscript: Pawan Pandeya, Durga Khadka Mishra, Shankar Prasad Khanal, Yadav Prasad Joshi, Sujan Babu Marahatta, Sushila Baral, Neha Deo, Santaram Chaudhary

Writing review and editing: Pawan Pandeya, Shankar Prasad Khanal, Sushila Baral

\section{DISCLOSURE STATEMENT}

The authors declare no possible conflicts of interest.

\section{FUNDING}

Funding for the study was received from University Grant Commission (UGC) Sanothimi, Bhaktapur in 2076-77.

\section{ACKNOWLEDGMENTS}

We would like to acknowledge all the workers and staffs of selected cement factories for their support during data collection.

\section{Ethical Approval: Approved}

\section{REFERENCES}

1. Joshi SK.Occupational Safety and Health in Nepal.Int J Occup Saf Health.2011 Aug 1; $1: 1-2$.

2. Kumar AS, Shrivastava SM, Patel P. Identification of Occupational diseases, Health risk, Hazard and Injuries among the workers engaged in Thermal Power Plant. International Journal of Research in Engineering and Technology. 2015; Vol 4 (1):149-156.

3. Ahmad I, Sattar A, Nawaz A. Occupational health and safety in industries in developing world. Gomal J Med Sci. 2016; 14:223-8.

4. G SK, M Z, Nair SB, $R$ VP. An epidemiological study on effect of occupational exposure of cement. International Journal of Community Medicine and Public Health. 2018 Nov 24;5(12):5105-9.

5. Quest Forum. A study report on Industrial accidents in cement industries of Nepal. 
Occupational safety and health project; 2017.

6. Pariyar SK., Das T, Ferdous T. Environment and Health Impact for Brick Kilns in Kathmandu Valley. International journal of Scientific and Technology Research. 2013; 2(5):184-187.

7. Mehraj. SS., Bhat G.A., Balkhi. H.M. Cement Factories and Human Health. Int $\mathbf{J}$ Cur Res Rev.2013 Sept; 05 (18): 47-53.

8. Mwaiselage J, Bratveit M, Moen BE, Mashalla Y. Respiratory symptoms and chronic obstructive pulmonary disease among cement factory workers. Scand J Work Environ Health 2005 Aug; 31(4):316323.

9. Ahmed HO, Newson-Smith MS. Knowledge and practices related to occupational hazards among cement workers in United Arab Emirates. J Egypt Public Health Assoc. 2010;85(3-4):149-67.

10. Department of Local Infrastructure Development and Agricultural Roads (DoLIDAR). Occupational safety and health guidelines. Ministry of Federal Affairs and Local Development: Government of Nepal; 2017. 76p.

11. Siyoum K, Alemu K, Kifle M. Respiratory Symptoms and Associated Factors among Cement Factory Workers and Civil Servants in North Shoa, Oromia Regional State, North West Ethiopia: Comparative CrossSectional Study. Occupational Medicine \& Health Affairs. 2014 Jan; 2:4.

12. Gautam R,Prasain J.Current situation of occupational safety and health in Nepal: A study report. General Federation of Nepalese Trade Unions: GEFONT publication; 2012. 96p.

13. Prasain, K. Domestic cement output continues to grow steadily. The Kathmandu Post. 2018.

14. Koirala MP. Health and Safety Concern of Workers of Building Materials Producing Industries in Nepal. IJERT. 2016 Dec 1; V5(12): IJERTV5IS120035.

15. Sah JP, Shah SK, Yadav DK, Salahuddin M, Yadav CK, Razin MS, et al. Knowledge and practice related to Occupational Hazards among Maruti Cement Factory workers in Mirchaiya, Siraha, Nepal. Microbes and Health. 2015;4(2):11-8.

16. Singh SB, Gautam S, Gautam R, Shrestha G, Jha N, Sharma SK. Respiratory Problems among Workers of Udayapur Cement
Factory in Eastern Nepal. Journal of Nepal Health Research Council. 2019 Apr 28;17(01):51-5.

17. Sana S, Bhat DGA, Balkhi HM. Health Risks Associated with Workers in Cement Factories. International Journal of Scientific and Research Publications .2013;3(5):5.

18. Workplace Safety and Prevention Services. Workplace inspection checklist. 2017:4p. Available from: https://www.wsps.ca/WSPS/media/Site/Res ources/SmallBusiness/sb_100-CCO-08-

IGDO_WorkplaceInspectionChecklist.pdf?e $\mathrm{xt}=$.pdf.

19. Kyaw MKK, O WM, Mya KM. Knowledge, Attitude and Practice on safety measures of occupational hazards among constructional workers at Bayint Naung Bridge Construction Site in Yangon Region. Myanmar Medical Journal, 2015;57(2):2832.

20. Gizaw Z, Yifred B, Tadesse T. Chronic respiratory symptoms and associated factors among cement factory workers in Dejen town, Amhara regional state, Ethiopia, 2015. Multidisciplinary Respiratory Medicine. 2016; 11:13.

21. Sundarraj A. The prevalence of respiratory morbidity and the risk factors associated, among the workers of cement industry in South India: A cross-sectional study [Dissertation submitted in partial fulfillment of the requirement for the award of the degree of Master of Public Health]. Sree Chitra Tirunal Institute for Medical Sciences and Technology Thiruvananthapuram, Kerala India; 2012. Available from: http://dspace.sctimst.ac.in/jspui/bitstream/12 3456789/2130/1/MPH_6147.pdf.

22. Ahmad MS, Sami W, Alharbi ME. WorkRelated Respiratory Complications among the Worker of Qassim Cement Industry of Qassim Region, Saudi Arabia. J Res Med Dent Sci. 2019; 7(6): 01-06.

23. Farooq I, Majeed A, Fatima N. Assessment of the Respiratory Symptoms among the Workers of Cement Factory. Indo Am. J. P. Sci, 2019; 06(03):6560-6577.

24. R. P, Clevin R, Ghattargi C, Dorle A, Lalitha D, Lalitha D. Effects of occupational dust exposure on the health status of portland cement factory workers. International Journal of Medicine and Public Health. 2013;3(3):192-6. 
Pawan Pandeya et.al. Occupational health risk among selected cement factory workers in dang district of Nepal.

25. Goldsheyder D, Weinera S S, and et.al. Musculoskeletal symptom survey among cement and concrete workers. IOS Press and the authors Work. 2004: 111-121.

26. Reddy GMM, Nisha B, Prabhushankar TG, Vishwambhar V. Musculoskeletal morbidity among construction workers: A crosssectional community-based study. Indian $\mathrm{J}$ Occup Environ Med. 2016;20(3):144-149. doi:10.4103/0019-5278.203134

27. Hussain AM, Hussain E, Azza E, Ahmed K, Mariam E. Risk factors associated with respiratory symptoms among cement workers. Poster session presented at: Chest
Annual Meeting. American college of chest physicians; 2019 Oct 19-23; America.

28. M.H. Karimfar, S. Shokri, M.R Gholami, A. Bayat, N. Moosavinasab and A. Choobineh. Musculoskeletal Problems among Workers of an Iranian Zinc Industry. Pakistan Journal of Biological Sciences. 2008;11: 2670-2674.

How to cite this article: Pandeya P, Mishra DK, Khana SP et.al. Occupational health risk among selected cement factory workers in dang district of Nepal. Int J Health Sci Res. 2021; 11(5):8-22. DOI: https://doi.org/10.52403/ijhsr.20210502 